\title{
Can Nidogen-1 and Nidogen-2 improve our preoperative cancer detection rate?
}

\author{
Agnieszka Kwiatkowska, ${ }^{1, A-F \oplus}$, Dominika Krawczyk ${ }^{2, A-B, D, F \oplus}{ }^{\text {, Krzysztof Kułak }}{ }^{1, C-F \oplus ~}$ \\ $17^{\text {st }}$ Chair and Department of Gynaecologial Oncology and Gynaecology, Medical University of Lublin, Poland \\ ${ }^{2}$ Medical University of Lublin, Poland \\ A - Research concept and design, B - Collection and/or assembly of data, C - Data analysis and interpretation, \\ $D$ - Writing the article, E - Critical revision of the article, F- Final approval of the article
}

Kwiatkowska A, Krawczyk D, Kułak K. Can Nidogen-1 and Nidogen-2 improve our preoperative cancer detection rate? J Pre-Clin Clin Res. 2021; 15(2): 80-86. doi: $10.26444 / j p c c r / 138308$

\begin{abstract}
I Abstract
Introduction and objective. Ovarian cancer $(\mathrm{OC})$ is the third most commonly diagnosed gynecological cancer among women worldwide and the second most common in Poland. Early-stage ovarian cancer is still very difficult to diagnose and concerns only about $20-30 \%$ of all ovarian cancers. Most cases (approximately $70 \%$ ) of ovarian cancer are diagnosed at more advanced stages (III and IV). The aim of the review is to bring closer new potential biological markers - Nidogen-1 and Nidogen- 2 in the diagnosis of ovarian cancer.

Brief description of the state of knowledge. To date, the best serum marker for ovarian cancer is Ca-125, but its use as a screening marker is limited due to high false positive rates. Ca-125 could be elevated in other benign and malignant conditions. Serum concentrations of Nidogen-1 and Nidogen-2 are higher in the advanced stagegroup (Stage III and IV), in comparison to the early stage group (Stage I and II) in serous ovarian cancer, and can reflect the tumour burden. Analysis showed that Nidogens discriminate against patients with serous ovarian carcinomas from healthy controls. The concentrations of both of them correlate with concentration Ca-125, especially Nidogen-2. The above biomarkers were compared with the results of the preoperative detection of ovarian cancer that are often used in clinical practice - IOTA Simple Rules, Risk of Malignancy Index and Risk of Ovarian Malignancy Algorithm.

Conclusions. Nidogen-1 and Nidogen-2 are new promising biomarkers for ovarian cancer, especially for the serous type, although there is still a need for prospective studies proving their good diagnostic value.
\end{abstract}

\section{Key words}

ovarian cancer, serous ovarian cancer, Nidogen-1, Nidogen-2

\section{INTRODUCTION}

Epithelial ovarian cancer is the most common type of ovarian cancer (OC), classified by several histological subtypes based on tumour cell morphology. The main histological subtypes of $\mathrm{OC}$ are serous, mucous, clear cell, and endometrioid. The most common is the epithelial serous type [1]. In cancer statistics the estimated number of new OC cases in the USA in 2020 was reported at 21,750 . In addition, they accounted for 13,940 estimated deaths for all types of OC in 2020 in the USA alone [2]. In the latest available statistics for Poland, 5,077 new cases of OC were diagnosed in 2018, and 3,204 women died from this disease [3]. Preoperative diagnostic tools detecting $\mathrm{OC}$ include: tumour imaging, measurement of serous antigens serum: CA-125 and HE4. OC is diagnosed by pathomorphological examination of tumour samples obtained from the primary surgery. New studies in recent years report some new promising developments regarding plasma levels of new biomarkers for ovarian serous cancer.

Address for corespondence: Agnieszka Kwiatkowska, lst $^{\text {st }}$ Chair and Department of Gynaecologial Oncology and Gynaecology, Medical University, Staszica 16, 20-081 Lublin, Poland.

E-mail: agakwiatkowska2604@gmail.com

Received: 04.03.2021; accepted: 31.05.2021; first published: 10.06.2021

\section{OBJECTIVE}

The aim of this review is to present the current knowledge of Nidogen- 1 and Nidogen- 2 and the possibility of using these proteins as screening tools for ovarian cancer. A literature review was conducted in the PubMed database, the terms: 'ovarian cancer,' 'CA-125, 'IOTA', 'RMI', 'ROMA', 'Nidogen-1', 'Nidogen-2' were searched.

Ovarian cancer preoperative detection - Carcinoma Antigen 125. Carcinoma Antigen 125 (CA-125) is routinely measured during the diagnosis and monitoring process of OC. CA-125 is produced physiologically by various cells in the body, including the endometrium, walls of the fallopian tubes and cervical mucosa. The upper limit for the serum CA-125 antigen is $35 \mathrm{U} / \mathrm{ml}$; however, greater specificity for proliferative changes is determined for values above $70 \mathrm{U} /$ $\mathrm{ml}$ [4]. This protein is characterized by a very high specificity and sensitivity in the detection of OC. In the case of OC, the values may reach several thousand, especially in the case of serous, endometrial and clear cell carcinomas. CA125 levels are increased in over $80 \%$ of women with OC, and only in less than $1 \%$ of healthy women [5]. CA-125 is elevated in approximately $50 \%$ of women with FIGO stage I OC. True positive results for FIGO stages II, III and IV are obtained in approximately $80 \%$ of women. Unfortunately, using only the CA-125 marker, the remaining percentage of OC would remain undetected. It is worth mentioning that the determination of CA-125 in patients with OC is 
more useful in post-surgical control, monitoring of adjuvant treatment (chemotherapy), prognosis and diagnosis of cancer recurrence [6] (Tab. 1).

International Ovarian Tumour Analysis (IOTA) - Simple Rules. In 2008, the International Ovarian Tumour Analysis (IOTA) proposed a new diagnostic method called 'Simple Rules' to distinguish benign from malignant ovarian masses [14]. In the available literature, the reports from the IOTA group are the largest reported study on ultrasound diagnosis of ovarian pathology [15]. The IOTA Simple Rules are intended to help distinguish the clinical features of malignancy from benignity during ultrasound examination [16]. These principles can be successfully applied by novice sonographers. The IOTA Simple Rules have created coherence in the definition of morphological features of ovarian tumours using a standardized examination technique (ultrasound features suggesting malignancy (M-features) or benignity (B-features)) [16,17] (Tab. 2, Tab. 3).

Risk of Malignancy Index. In 1990, Jacobs et al. suggested for the first time distinguishing a benign from a malignant pelvic mass [17]. The Risk of Malignancy Index (RMI) model includes clinical data, biochemical results and sonographic findings of women with suspected malignant changes of the ovary.
The RMI is calculated by the formula $-\mathrm{RMI}=\mathrm{U} \times \mathrm{M} \times \mathrm{Ca}-125$ where: $U=$ ultrasound score, $M=$ menopause status $(M=1$ for pre-menopausal women, $\mathrm{M}=3$ for post-menopausal women). The cut-off value was 200 , which correlated with a high probability of malignancy [23]. Over the years, the RMI model has evolved and three modifications have been developed depending on the values assigned to the $U$ and $M$ features [24]. This model allows separation of patients with benign lesions. Unfortunately, this algorithm does not qualify patients according to the FIGO clinical stage $[25,26,27,28]$.

Calculation of specificity, sensitivity, positive and negative predictive values is not based on the value of the result, but on its value below or above this threshold $(200)[4,17]$. (Table 4)

Risk of Ovarian Malignancy Algorithm. The Risk of Ovarian Malignancy (ROMA) algorithm uses a combination of cancer antigen 125 (CA-125), human epididymal protein 4 (HE4) and women's menopausal status. ROMA allows assessment of the degree of risk and assignment of women with adnexal/ small pelvis masses to groups with low or high risk of an ovarian malignancy. The cut-off points are, respectively: for premenopausal women $>13.15$ and for postmenopausal women $\geq 27.7[30,31,32]$. The ROMA algorithm has not been validated for women with previous oncological history,

Table 1. Diagnostic performance of CA 125 in the subset of studies to date

\begin{tabular}{|c|c|c|c|c|c|c|}
\hline \multirow[b]{2}{*}{ Author } & \multicolumn{6}{|c|}{ CA 125} \\
\hline & Systematic review or meta-analysis & SN \% (95\% IC) & SP \% (95\% IC) & PPV \% & NPV \% & AUC $(95 \%$ IC) \\
\hline Huy et al.* [5] & & 92.3 & 77.0 & 19.05 & 99.40 & 0.872 \\
\hline Huy et al. ${ }^{\circ}[5]$ & & 76.5 & 90.0 & 81.25 & 87.10 & 0.872 \\
\hline Lee et al.* [6] & & 58.4 & 55.6 & 17.5 & 89.3 & 0.56 \\
\hline Lee et al. ${ }^{\circ}[6]$ & & 57.8 & 88.3 & 62.7 & 86 & 0.806 \\
\hline Yanaranop et al. ${ }^{\circ}[7]$ & & 80.4 & 69.7 & 64.9 & 83.6 & \\
\hline Goff et al. ${ }^{\varepsilon}[8]$ & & $79(67-88)$ & $76(68-83)$ & 63 & 87 & \\
\hline Shin et al.* [9] & & 52.6 & 70.1 & & & 0.569 \\
\hline Shin et al. ${ }^{\circ}[9]$ & & 90.0 & 85.7 & & & 0.917 \\
\hline Lycke et al.* [10] & & 95.7 & 59.6 & 19.1 & 99.3 & $0.776(0.723-0.829)$ \\
\hline Kim et al.* [11] & & 71.4 & 69.5 & 5.5 & 99 & \\
\hline Kim et al. ${ }^{\circ}[11]$ & & 71.4 & 90.3 & 67.8 & 91 & \\
\hline Choi et al.* [12] & & 74.7 & 78.7 & & 91 & 0.777 \\
\hline Choi et al..$^{\circ}[12]$ & & 82.1 & 94.9 & & 62.2 & 0.852 \\
\hline Torky et al. ${ }^{\varepsilon}[13]$ & & 92.8 & 68.9 & 41.9 & 97.2 & 0.736 \\
\hline
\end{tabular}

* premenopausal subgroup; ${ }^{\circ}$ postmenopausal subgroup; ${ }^{\varepsilon}$ - combined (premenopausal and postmenopausal) subgroup

SN - Sensitivity; SP - Specificity; PPV - Positive Predictive Value; NPV - Negative Predictive value; AUC - Area Under ROC Curve;

Table 2. IOTA Simple Rules for predicting benign or malignant ovarian tumour $[14,16]$

\begin{tabular}{ll}
\hline $\begin{array}{l}\text { Rules for predicting a malignant tumour } \\
\text { (M-rules) }\end{array}$ & $\begin{array}{l}\text { Rules for predicting a benign tumour } \\
\text { (B-rules) }\end{array}$ \\
\hline M1 Irregular solid tumour & B1 Unilocular cyst \\
\hline M2 Presence of ascites & $\begin{array}{l}\text { B2 Presence of solid components where the largest solid component is }<7 \text { mm in largest } \\
\text { diameter; }\end{array}$ \\
\hline M3 At least four papillary structures & B3 Presence of acoustic shadows \\
\hline M4 Irregular multilocular solid tumour with largest diameter $\geq 100 \mathrm{~mm}$ & B4 Smooth multilocular tumour with largest diameter $<100 \mathrm{~mm}$ \\
\hline M5 Very strong blood flow (color score 4) & B5 No blood flow (colour score 1)
\end{tabular}


women currently undergoing chemotherapy, pregnant women or young women (under 18 years of age) $[31,32]$. The test is not intended as a screening test or a standalone diagnostic test for ovarian cancer $[10,31]$. (Table 5)

What are Nidogen-1 and Nidogen-2? Human body cells are divided into specialized groups by a thin membranous layer of connective tissue called the basement membrane, and links body cells to their interstitial matrix [34]. The most important parts of the basement membrane are laminins, collagen IV, nidogens and perlecan. Nidogens connect proteins from laminin networks and collagen IV. This process supports stabilization of the structure created by the basement membrane [35,36]. Nidogen-1 (NID-1) and Nidogen-2 (NID2) are in a group of nidogens that are observed in human organisms [34].

Both nidogens are made up of three globular domains which are divided by a region resembling a link or a rod [37]. Mesenchymal cells indicate the presence of nidogens which are collected in the endothelial and epithelial basement membranes within the phase of development [38].
Further, fibroblasts are classified as an origin of nidogens in skin tissue $[38,39]$. Moreover, the nidogens described above have been observed in human perlecan [40,41].

Both NID-1 and NID-2 have the ability to combine with various receptors in human cells. NID-1 concentrates in cartilage tissue and neuromuscular junction, whereas NID-2 is manifested in muscle tissue [42]. Additionally, damage to NID-1 in mouse cells resulted in anionic modification in the glomerular basement membrane and neurological failures, such as structural changes in capillaries in the brain and the capsule of the lens. Moreover, disturbance to wound healing was also observed [43]. Both nidogens are evidently likely to cleave by proteolysis; nevertheless, laminin c1-binding may reduce that tendency for NID-1 [38]. What is more, that effect was also observed between nidogens, thus extraction of both NID-1 and NID-2 may results in destruction of the basement membrane $[39,44,45]$. NID-2 might also be useful as a biomarker to predict non-small cell lung cancer (NSCLC) or to screen the high-risk population for NSCLC, and additionally for screening for esophageal squamous cell carcinoma $[46,47,48]$.

Table 3. Diagnostic performance of IOTA Simple Rules algorithm in the subset of studies to date

\begin{tabular}{|c|c|c|c|c|c|c|}
\hline \multirow[b]{2}{*}{ Author } & \multicolumn{6}{|c|}{ IOTA Simple Rules } \\
\hline & Systematic review or meta-analysis & SN \% (95\% IC) & SP $\%(95 \%$ IC) & PPV \% & NPV \% & $\operatorname{AUC}(95 \% \mathrm{IC})$ \\
\hline Auekitrungrueng et al. $\varepsilon[15]$ & & $83.8(77.1-90.4)$ & $92(88.8-95.2)$ & $\begin{array}{c}81.7 \\
(74.7-88.6)\end{array}$ & $\begin{array}{c}93 \\
(88.5-97.6)\end{array}$ & \\
\hline Garg S et al.e[16] & & 91.66 & 84.84 & 68.75 & 96.55 & 0.889 \\
\hline Tongsong et al. $\varepsilon$ [18] & & 82.9 & 94 & & & \\
\hline Ning et al. $\varepsilon[19]$ & & 96.2 & 96.3 & 92.7 & 98.1 & 0.96 \\
\hline Ning et al.e [19] & & 72.4 & 88.8 & 77.8 & 85.6 & 0.86 \\
\hline Ning et al.e [19] & & 100 & 94 & 90.5 & 100 & 0.97 \\
\hline Ning et al. $\varepsilon$ [19] & & 96.7 & 87.3 & 81.7 & 97.8 & 0.92 \\
\hline Knafel et al. $\varepsilon[20]$ & & 96.3 & 81.9 & 87.2 & 75.2 & \\
\hline Knafel et al. $\varepsilon[20]$ & & 96.3 & 95.1 & 91.9 & 97.9 & \\
\hline Knafel et al.e [20] & & 95.1 & 89.6 & 83.9 & 97 & \\
\hline Knafel et al. $\varepsilon[20]$ & & 95.1 & 93.8 & 89.7 & 97.1 & \\
\hline Shetty et al. $\varepsilon[21]$ & & $92.8(77-99)$ & $92.9(88-96.4)$ & $70.2(53-84)$ & $98.6(95-99)$ & \\
\hline Hidalgo et al. $\varepsilon[22]$ & & 95.6 & 97.8 & & & \\
\hline Hidalgo et al. $\varepsilon[22]$ & & 94.1 & 97.6 & & & \\
\hline
\end{tabular}

* premenopausal subgroup; ${ }^{\circ}$ postmenopausal subgroup; $\varepsilon$ - combined (premenopausal and postmenopausal) subgroup;

SN - Sensitivity; SP - Specificity; PPV - Positive Predictive Value; NPV - Negative Predictive Value; AUC - Area Under ROC Curve;

Table 4. Diagnostic performance of RMI algorithm in the subset of studies to-date

\begin{tabular}{|c|c|c|c|c|c|c|}
\hline \multirow[b]{2}{*}{ Author } & \multicolumn{6}{|c|}{ RMI } \\
\hline & Systematic review or meta-analysis & SN \% (95\% IC) & SP \% (95\% IC) & PPV \% & NPV \% & AUC (95\% IC) \\
\hline Yanaranop et al.* [7] & & 75 & 80.8 & 47.7 & 93.3 & \\
\hline Yanaranop et al. ${ }^{\circ}[7]$ & & 80.4 & 77.3 & 71.2 & 85.0 & \\
\hline Lycke et al.* [10] & & 87.0 & 89.6 & 45.5 & 98.6 & $0.883(0.810-0.956)$ \\
\hline Lycke et al. ${ }^{\circ}[10]$ & & 89.3 & 80.5 & 70.4 & 93.5 & $0.849(0.810-0.888)$ \\
\hline Auekitrungrueng et al..$^{\varepsilon}[15]$ & & $77.2(70.4-84.1)$ & $86.8(83.2-90.5)$ & $71.8(64.7-78.9)$ & $89.8(85.0-94.5)$ & \\
\hline Auekitrungrueng et al. $[15]$ & & $82.1(75.8-88.3)$ & $82.6(78.6-86.7)$ & $67.2(60.3-74.1)$ & $91.4(87.3-95.5)$ & \\
\hline Meys et al. ${ }^{\varepsilon}[29]$ & $x$ & 75 (72-79) & $92(88-94)$ & & & \\
\hline Chacón et al. ${ }^{8}[30]$ & $\mathrm{x}$ & 87 & 75 & & & \\
\hline
\end{tabular}

* premenopausal subgroup; ${ }^{\circ}$ postmenopausal subgroup; ${ }^{\varepsilon}$ - combined (premenopausal and postmenopausal) subgroup

SN - Sensitivity; SP - Specificity; PPV - Positive Predictive Value; NPV - Negative Predictive Value; AUC - Area Under ROC Curve 
Table 5. Diagnostic performance of ROMA algorithm in the subset of studies to date

\begin{tabular}{|c|c|c|c|c|c|c|}
\hline \multirow[b]{2}{*}{ Author } & \multicolumn{6}{|c|}{ ROMA } \\
\hline & Systematic review or meta-analysis & SN \% (95\% IC) & SP \% (95\% IC) & PPV \% & NPV \% & AUC $(95 \%$ IC) \\
\hline Huy et al.* [5] & & 76.9 & 82.9 & 21.28 & 98.36 & 0.912 \\
\hline Huy et al. ${ }^{\circ}[5]$ & & 82.4 & 96.7 & 93.33 & 90.62 & 0.912 \\
\hline Lee et al.* [6] & & 45.5 & 81.8 & 28.8 & 90.3 & 0.768 \\
\hline Lee et al..$^{\circ}[6]$ & & 50 & 88.8 & 60.4 & 83.9 & 0.79 \\
\hline Yanaranop et al. ${ }^{\circ}[7]$ & & 82.6 & 65.2 & 62.3 & 84.3 & \\
\hline Shin et al.* [9] & & 52.6 & 87.9 & & & 0.792 \\
\hline Shin et al. ${ }^{\circ}[9]$ & & 95.0 & 87.1 & & & 0.980 \\
\hline Lycke et al.* [10] & & 87.0 & 80.9 & 31.3 & 98.4 & $0.839(0.764)$ \\
\hline Lycke et al. ${ }^{\circ}[10]$ & & 91.1 & 77.2 & 67.5 & 94.3 & $0.841(0.803-0.880)$ \\
\hline Kim B et al. ${ }^{\circ}[11]$ & & 69.6 & 91.3 & 69.6 & 91.3 & \\
\hline Choi et al.*[12] ${ }^{12}$ & & 70.7 & 92.6 & & 91.1 & 0.875 \\
\hline Choi et al..$^{\circ}[12]$ & & 82.9 & 97.4 & & 63.9 & 0.864 \\
\hline Chacón et al.e [30] & $x$ & 77 & 85 & & & \\
\hline Cui et al. $\varepsilon[32]$ & $x$ & $90(88-93)$ & $91(89-94)$ & $90(88-95)$ & $93(91-95)$ & 0.96 \\
\hline Abdalla et al.* [33] & & $70(41.6-98.4)$ & $82(76.4-87.7)$ & $17.9(5.9-30)$ & $98(95.7-100)$ & $0.814(0.758-0.869)$ \\
\hline Abdalla $\mathrm{N}$ et al. ${ }^{\circ}[33]$ & & 82.5 (70.7-94.3) & $83.8(75.4-92.2)$ & $73.3(60.4-86.3)$ & 89.9 (82.7-97) & $0.833(0.765-902)$ \\
\hline
\end{tabular}

* premenopausal subgroup; ${ }^{\circ}$ postmenopausal subgroup; $\varepsilon$ - combined (premenopausal and postmenopausal) subgroup

SN - Sensitivity; SP - Specificity; PPV- Positive Predictive Value; NPV- Negative Predictive Value; AUC - Area Under ROC Curve

Despite the protective function of nidogens for the basement membrane, they have potential to be a target for destruction of tissues during carcinogenesis, metastasis formatting and angiogenesis stimulation, which might explain the promoting impact for carcinogenesis of the stomach or colon triggered by nidogens loss [46].

\section{DISCUSSION}

Nidogen-1 in ovarian cancer. Several authors from Beijing checked the level of NID-1 and CA-125 in ovarian tissues from 265 patients with serous carcinoma (OSC). The results of the study indicated a high level of NID-1 in advanced stage cells (stage III and IV), compared to the early stages (stage I and II) [49]. In their analyses, they also took into consideration the plasma concentration of the protein in cell samples obtained from OSC patients. They found that the levels of NID-1 were noticeably increased in the examined tissues in opposition to normal cells. It was observed, however, that CA-125 rather than NID-1 may be more useful in OC patients in the early stages of the disease (CA-125: 0.88, 95\% Cl, 0.80-0.96, NID-1: $0.53,95 \% \mathrm{Cl}, 0.41-0.64$ ) [49].

Statistical analysis shows that NID-1 has a potential value to predict certain aspects of responses to treatment. The levels of NID-1 were compared in two groups of women: 138 patients with primary inoperable cancerous tumour after chemotherapy, and 127 patients who underwent surgical macroscopic cytoreduction of the neoplasm. Interestingly, in patients after chemotherapy, the levels of NID-1 and CA125 were higher than in those who underwent surgery alone. These results indicate that the level of NID-1 may predict the volume of tumour burden and the most appropriate and individually tailored therapeutic path.
Moreover, it was observed that the levels of both NID-1 and CA-125 correlated with grading of OC and its sensitivity to chemotherapy. On the other hand, no correlation was found between nidogen-1 and metastases in lymph nodes, in opposition to CA-125 [49].

Additionally, a study by Li-Zhang et al. examined the expression of NID-1 in the tissues from 44 patients with ovarian serous carcinoma (OSC). The protein was detected in intracellular substance and cytoplasm. It is worth noting that in the group of patients with OSC, the expression of NID-1 in the analyzed tissues was not related to progression of the tumour [49]. Interestingly, some authors concluded that there was a significant relationship between the level of NID-1 and poor clinical prognosis in patients with OSC. A study by Zhou et al. revealed that NID-1 propagates the epithelial-to-mesenchymal transition (EMT) of ovarian tissue in serous cancer. Additionally, under the influence of NID-1 the tissue may change its epithelial features into mesenchymal attributes. NID-1 supports migration, penetration, and decreases the sensitivity of ovarian tissue to OC. The regulation uses the mechanism of initiation of the ERK/MAPK pathway [50].

On the basis of the study by Yuan et al., it was concluded that NID-1 became a direct target of miR-204-3p. LncRNAATB controls miR-204-3p in a negative way. Through NID-1 over-expression, LncRNA-ATB, which promotes apoptosis and inhibits proliferation of ovarian cancer cells, may be inhibited and will stimulate the growth of OC cells [51]. Furthermore, Zhou et al. reported the impact of NID-1 on the loss of sensitivity to chemotherapy based on cisplatin in ovarian cancer. Among the examined neoplastic tissues, the levels of NID-1 correlated with higher resistance to cisplatin-based chemotherapy [50]; therefore, NID-1 may represent a candidate prognostic indicator and a potential therapeutic target in OC. 
Nidogen-2 in ovarian cancer. In the research by Kuk et al., the levels of NID-2 and CA-125 were compared in three groups of women. The groups included a control group, a group with benign ovarian pathologies, and a group with ovarian cancer (FIGO stage I/II and III/IV). Each group comprised 100 patients. The study revealed that the level of NID-2 in the serum of patients with OC was elevated (median $18.6 \mu \mathrm{g} / \mathrm{L}$ ), compared to patients with benign ovarian pathologies and the control group (median 12.1 and $13.2 \mu \mathrm{g} / \mathrm{L}$, respectively) [52].

Analysis showed that NID-2 has a potential statistical value. The results revealed that NID-2 strongly correlates with the values of CA-125. Both CA-125 and NID-2 are more often elevated in the serous type compared to the mucinous, endometrioid or clear cell type of ovarian cancer. Serum NID-2 is also more frequently elevated at the latestage disease (FIGO stage III/IV), similar to CA-125 [52]. Several other potential biomarkers were also determined; however, most of them are not clinically relevant in the prediction of ovarian cancer $[53,54]$. It was the first study measuring the level of NID-2 and showing its correlations with CA-125. It demonstrated possible clinical benefits in OC prophylaxis, after prior extensive proteomic analysis of OC ascites, identifying over 450 proteins [55].

Torky et al., assessed in their study the levels of NID-2 and CA-125 in the serum of OC patients, and the value of transvaginal ultrasonography in the prediction of the disease. The study group comprised 144 women with an adnexal mass detected, including 116 benign and 28 malignant cases. In histopathological examination of the benign and malignant cases, CA-125 and NID-2 serum concentrations were similarly distributed. In this study, the cut-off value for NID-2 was $28.35 \mathrm{ng} / \mathrm{ml}$, which showed $91.6 \%$ sensitivity, $62 \%$ specificity, PPV $37.1 \%$, NPV $97.9 \%$ and $68 \%$ accuracy [13]. The study showed that the combination of more than one marker is much more beneficial in OC prediction. Low sensitivity of transvaginal ultrasonography is augmented by a much higher sensitivity of tumour markers, while in the opposite situation, Doppler ultrasonography (U/S) corrects low results of tumour markers [13].

The study by Chen et al. concerned an increase in NID-2 and CA-125 levels in patients with serous OC. The levels of NID-2 and CA-125 were examined by the ELISA method and immunoassay in 15 patients in the control group, 22 patients with benign ovarian pathologies and 40 patients with serous OC [56]. Comparing the obtained results, it was found that the level of NID-2 in the control group and in patients with benign ovarian pathologies was significantly lower compared to patients with serous OC. The results correlated with the conclusions of Kuk and Torky in their studies. The differences in NID-2 levels between the control group and patients with benign ovarian pathologies were not significant. In the serous type of OC the level of serum NID-2 was higher in patients at stages III-IV than in patients at stages I-II. Diagnostic specificity and sensitivity were improved due to the combined detection of serum NID-2 and CA-125. The study showed that NID-2 could not only be used as a new biomarker, but also as a prognostic marker to assess progression of ovarian cancer [56].

Several diagnostic biomarkers can be combined in order to increase sensitivity and specificity of tests in the detection of ovarian cancer. Such studies have already been conducted. Comparing NID-2 values with CA-125 values, transvaginal ultrasonography and currently used preoperative methods in ovarian cancer, it can be seen that NID-2 is more sensitive than Doppler U/S, CA-125, RMI and ROMA, and slightly less sensitive than the IOTA Simple Rules. NID-2 specificity, PPV and accuracy are lower than all the above- mentioned methods, but NPV is the highest compared to CA-125, Doppler U/S, RMI, ROMA and the IOTA Simple Rules.

\section{CONCLUSIONS}

To-date, no effective screening methods have been developed for decreasing the incidence of ovarian cancer and its its high mortality. Effective biomarkers for early detection of this malignancy are needed. Diagnostic markers and indicators are desirable - sensitive and very specific for a given histological type of ovarian cancer. In the future, NID-1 and NID-2 may be useful in early diagnostics of ovarian cancer, especially its serous type. Their increased concentration suggests adenocarcinoma of the ovary, both at the early and late stages. A decrease in their concentration may suggest growth of the stomach and colon tumours. There is still a need for prospective studies combining the diagnostic value of NID-1, NID-2 and current ovarian cancer diagnostic algorithms in order to check their diagnostic and predictive value. Close correlation between CA-125 and NID2 prevents their combination in one diagnostic panel which would perform better than CA-125 alone. However, NID-2 seems to be a promising biomarker that correlates closely with ultrasound and CA-125. Although it has improved the accuracy of diagnosis, further studies are still needed to validate the described biomarkers.

\section{Acknowledgements}

The authors gratefully acknowledge the multiple databases from which the data was obtained.

\section{Disclosure statement, author contributions, funding}

No potential conflict of interest was reported by the author(s). The article is an original work, has not been published previously, and is not under consideration for publication elsewhere in its final form - printed or electronic. All authors participated in the conception and design of the study; all authors approved the final manuscript as submitted, and agree to be accountable for all aspects of the study.

The study did not receive any grant from funding agencies in the public, commercial, or not-for-profit sectors.

\section{REFERENCES}

1. Tan TZ, Miow QH, Miki Y, et al. Epithelial-mesenchymal transition spectrum quantification and its efficacy in deciphering survival and drug responses of cancer patients. EMBO Mol Med. 2014; 6(10): 12791293. doi: 10.15252/emmm.201404208

2. Siegel RL, Miller KD, Jemal A. Cancer statistics. CA: A Cancer Journal for Clinicians. 2020; 70(1): 7-30. doi: 10.3322/caac.21590

3. Globocan. Summary statistic 2018. Males. Females. Both sexes. 2018. Available from: https://gco.iarc.fr/today/data/factsheets/ populations/616-poland-fact-sheets.pdf

4. Dochez V, Caillon H, Vaucel E, et al. Biomarkers and algorithms for diagnosis of ovarian cancer: CA125, HE4, RMI and ROMA, a review. J Ovarian Res. 2019; 12(1): 28. doi: 10.1186/s13048-019-0503-7

5. Huy NVQ, Van Khoa V, Tam LM, et al. Standard and optimal cut-off values of serum ca-125, HE4 and ROMA in preoperative prediction of 
ovarian cancer in Vietnam. Gynecol Oncol Rep. 2018; 25: 110-114. doi: 10.1016/j.gore.2018.07.002

6. Lee YJ, Kim YM, Kang JS, et al. Comparison of Risk of Ovarian Malignancy Algorithm and cancer antigen 125 to discriminate between benign ovarian tumour and early-stage ovarian cancer according to imaging tumour subtypes. Oncol Lett. 2020; 20(1): 931-938. doi: 10.3892/ol.2020.11629

7. Yanaranop M, Anakrat V, Siricharoenthai S, et al. Is the Risk of Ovarian Malignancy Algorithm Better Than Other Tests for Predicting Ovarian Malignancy in Women with Pelvic Masses? Gynecol Obstet Invest. 2017; 82(1): 47-53. doi: 10.1159/000446238

8. Goff BA, Agnew K, Neradilek MB, et al. Combining a symptom index, CA125 and HE4 (triple screen) to detect ovarian cancer in women with a pelvic mass. Gynecol Oncol. 2017; 147(2): 291-295. doi: 10.1016/j. ygyno.2017.08.020

9. Shin KH, Kim HH, Kwon BS, et al. Clinical Usefulness of Cancer Antigen (CA) 125, Human Epididymis 4, and CA72-4 Levels and Risk of Ovarian Malignancy Algorithm Values for Diagnosing Ovarian Tumours in Korean Patients With and Without Endometriosis. Ann Lab Med. 2020; 40(1): 40-47. doi: 10.3343/alm.2020.40.1.40

10. Lycke M, Kristjansdottir B, Sundfeldt K. A multicenter clinical trial validating the performance of $\mathrm{HE} 4, \mathrm{CA} 125$, risk of ovarian malignancy algorithm and risk of malignancy index. Gynecol Oncol. 2018; 151(1): 159-165. doi: 10.1016/j.ygyno.2018.08.025

11. Kim B, Park Y, Kim B, et al. Diagnostic performance of CA 125, HE4, and risk of Ovarian Malignancy Algorithm for ovarian cancer. J Clin Lab Anal. 2019; 33(1): e22624. doi: 10.1002/jcla.22624

12. Choi HJ, Lee YY, Sohn I, et al. Comparison of CA 125 alone and risk of ovarian malignancy algorithm (ROMA) in patients with adnexal mass: A multicenter study. Curr Probl Cancer. 2020; 44(2): 100508. doi: 10.1016/j.currproblcancer.2019.100508

13. Torky HA, Sherif A, Abo-Louz A, et al. Evaluation of Serum Nidogen-2 as a Screening and Diagnostic Tool for Ovarian Cancer. Gynecol Obstet Invest. 2018; 83: 461-465. doi: 10.1159/000481798

14. Timmerman D, Van Calster B, Testa A, et al. Predicting the risk of malignancy in adnexal masses based on the Simple Rules from the International Ovarian Tumour Analysis group. Am J Obstet Gynecol. 2016 Apr; 214(4): 424-437. doi: 10.1016/j.ajog.2016.01.007

15. Auekitrungrueng R, Tinnangwattana D, Tantipalakorn C, et al. Comparison of the diagnostic accuracy of International Ovarian Tumour Analysis simple rules and the risk of malignancy index to discriminate between benign and malignant adnexal masses. Int J Gynaecol Obstet. 2019; 146(3): 364-369. doi: 10.1002/ijgo.12891

16. Garg S, Kaur A, Mohi JK, et al. Evaluation of IOTA Simple Ultrasound Rules to Distinguish Benign and Malignant Ovarian Tumours. J Clin Diagn Res. 2017; 11(8): TC06-TC09. doi: 10.7860/JCDR/2017/26790.10353

17. Antovska V, Trajanova M. An original risk of ovarian malignancy index and its predictive value in evaluating the nature of ovarian tumour. Southern African Journal of Gynaecological Oncology. 2015; 7(2): 52-59. doi: 10.1080/20742835.2015.1081486

18. Tongsong T, Tinnangwattana D, Vichak-Ururote L, et al. Comparison of Effectiveness in Differentiating Benign from Malignant Ovarian Masses between IOTA Simple Rules and Subjective Sonographic Assessment. Asian Pac J Cancer Prev. 2016; 17(9): 4377-4380.

19. Ning CP, Ji X, Wang HQ, et al. Association between the sonographer's experience and diagnostic performance of IOTA simple rules. World J Surg Oncol. 2018; 16(1): 179. doi: 10.1186/s12957-018-1479-2

20. Knafel A, Banas T, Nocun A, et al. The Prospective External Validation of International Ovarian Tumour Analysis (IOTA) Simple Rules in the Hands of Level I and II Examiners. Ultraschall Med. 2016; 37(5): 516-523. English. doi: 10.1055/s-0034-1398773

21. Shetty J, Saradha A, Pandey D, et al. IOTA Simple Ultrasound Rules for Triage of Adnexal Mass: Experience from South India. J Obstet Gynaecol India. 2019; 69(4): 356-362. doi: 10.1007/s13224-019-01229-z

22. Hidalgo JJ, Ros F, Aubá M, et al. Prospective external validation of IOTA three-step strategy for characterizing and classifying adnexal masses and retrospective assessment of alternative two-step strategy using simple-rules risk. Ultrasound Obstet Gynecol. 2019; 53(5): 693-700. doi: $10.1002 /$ uog. 20163

23. Al-Musalhi K, Al-Kindi M, Ramadhan F, et al. Validity of Cancer Antigen-125 (CA-125) and Risk of Malignancy Index (RMI) in the Diagnosis of Ovarian Cancer. Oman Med J. 2015; 30(6): 428-434. doi: 10.5001/omj.2015.85

24. Dora SK, Dandapat AB, Pande B, Hota JP. A prospective study to evaluate the risk malignancy index and its diagnostic implication in patients with suspected ovarian mass. J Ovarian Res. 2017; 10(1): 55. doi: 10.1186/s13048-017-0351-2
25. Chopra S, Vaishya R, Kaur J. An Evaluation of the Applicability of the Risk of Malignancy Index for Adnexal Masses to Patients Seen at a Tertiary Hospital in Chandigarh, India. J Obstet Gynaecol India. 2015; 65(6): 405-410. doi: 10.1007/s13224-014-0583-7

26. Campos C, Sarian LO, Jales RM, et al. Performance of the Risk of Malignancy Index for Discriminating Malignant Tumours in Women With Adnexal Masses. J Ultrasound Med. 2016; 35(1): 143-152. doi: 10.7863/ultra.15.01068

27. Anton C, Carvalho FM, Oliveira EI, et al. A comparison of CA125, HE4, risk ovarian malignancy algorithm (ROMA), and risk malignancy index (RMI) for the classification of ovarian masses. Clinics (Sao Paulo). 2012; 67(5): 437-441. doi: 10.6061/clinics/2012(05)06

28. Manegold-Brauer G, Buechel J, Knipprath-Mészaros A, et al. Improved Detection Rate of Ovarian Cancer Using a 2-Step Triage Model of the Risk of Malignancy Index and Expert Sonography in an Outpatient Screening Setting. Int J Gynecol Cancer. 2016; 26(6): 1062-1069. doi: 10.1097/IGC.0000000000000718

29. Meys EM, Kaijser J, Kruitwagen RF, et al. Subjective assessment versus ultrasound models to diagnose ovarian cancer: A systematic review and meta-analysis. Eur J Cancer. 2016; 58: 17-29. doi: 10.1016/j. ejca.2016.01.007

30. Chacón E, Dasí J, Caballero C, et al. Risk of Ovarian Malignancy Algorithm versus Risk Malignancy Index-I for Preoperative Assessment of Adnexal Masses: A Systematic Review and Meta-Analysis. Gynecol Obstet Invest. 2019; 84(6): 591-598. doi: 10.1159/000501681

31. Wei SU, Li H, Zhang B. The diagnostic value of serum HE4 and CA-125 and ROMA index in ovarian cancer. Biomed Rep. 2016 Jul; 5(1): 41-44. doi: 10.3892/br.2016.682

32. Cui R, Wang Y, Li Y, et al. Clinical value of ROMA index in diagnosis of ovarian cancer: meta-analysis. Cancer Manag Res. 2019; 11: 2545-2551. doi: 10.2147/CMAR.S199400

33. Abdalla N, Piorkowski R, Bachanek M, et al. Does the Risk of Ovarian Malignancy Algorithm Provide Better Diagnostic Performance Than HE4 and CA125 in the Presurgical Differentiation of Adnexal Tumours in Polish Women? Dis Markers. 2018: 5289804. doi: 10.1155/2018/5289804

34. Kruegel J, Miosge N. Basement membrane components are key players in specialized extracellular matrices. Cell Mol Life Sci. 2010; 67(17): 2879-2895. doi: 10.1007/s00018-010-0367-x

35. Dai J, Estrada B, Jacobs S, et al. Dissection of Nidogen function in Drosophila reveals tissue-specific mechanisms of basement membrane assembly. PLoS Genet. 2018; 14(9): e1007483. doi: 10.1371/journal. pgen.1007483

36. Miosge N, Sasaki T, Timpl R. Evidence of nidogen-2 compensation for nidogen-1 deficiency in transgenic mice. Matrix Biol. 2002; 21(7): 611-621. doi: 10.1016/s0945-053x(02)00070-7

37. Chai AW, Cheung AK, Dai W, et al. Metastasis-suppressing NID2, an epigenetically-silenced gene, in the pathogenesis of nasopharyngeal carcinoma and esophageal squamous cell carcinoma. Oncotarget. 2016 Nov 29; 7(48): 78859-78871. doi: 10.18632/oncotarget.12889

38. Patel TR, Bernards C, Meier M, et al. Structural elucidation of fulllength nidogen and the laminin-nidogen complex in solution. Matrix Biol. 2014; 33: 60-7. doi: 10.1016/j.matbio.2013.07.009

39. Lakshmanan HHS, Melrose AR, Sepp AI, et al. The basement membrane protein nidogen-1 supports platelet adhesion and activation. Platelets. 2020; 1: 1-5. doi: 10.1080/09537104.2020.1745170

40. Breitkreutz D, Koxholt I, Thiemann K, et al. Skin Basement Membrane: The Foundation of Epidermal Integrity-BM Functions and Diverse Roles of Bridging Molecules Nidogen and Perlecan. BioMed Research International. 2013; 1-16. doi: 10.1155/2013/179784

41. Saikia P, Thangavadivel S, Medeiros CS, et al. IL-1 and TGF- $\beta$ Modulation of Epithelial Basement Membrane Components Perlecan and Nidogen Production by Corneal Stromal Cells. Invest Ophthalmol Vis Sci. 2018 Nov 1; 59(13): 5589-5598. doi: 10.1167/iovs.18-25202

42. Meyer IFG. Investigating the role of nidogens, a family of basement membrane proteins, at the neuromuscular junction in health and disease. https://discovery.ucl.ac.uk/id/eprint/10075381/1/Final\%20 Thesis.pdf (access 2021.03.12).

43. Baranowsky A, Mokkapati S, Bechtel M, et al. Impaired wound healing in mice lacking the basement membrane protein nidogen 1. Matrix Biol. 2010; 29(1): 15-21. doi: 10.1016/j.matbio.2009.09.004

44. Strelnikov VV, Kuznetsova EB, Tanas AS, et al. Abnormal promoter DNA hypermethylation of the integrin, nidogen, and dystroglycan genes in breast cancer. Sci Rep. 2021; 11: 2264. doi: 10.1038/s41598-021-81851-y 45. Lössl P, Kölbel K, Tänzler D, et al. Analysis of nidogen-1/laminin $\gamma 1$ interaction by cross-linking, mass spectrometry, and computational modeling reveals multiple binding modes. PLoS One. 2014; 9(11): e112886. doi: 10.1371/journal.pone.0112886 
46. Yu ZH, Wang YM, Jiang YZ, et al. NID2 can serve as a potential prognosis prediction biomarker and promotes the invasion and migration of gastric cancer. Pathol Res Pract. 2019; 215(10): 152553. doi: $10.1016 / j . p r p .2019 .152553$

47. Feng X, Xie X, Zheng B, et al. The more potential performance of nidogen 2 methylation by tissue or plasma DNA over brichoalveolar lavage DNA in diagnosis of nonsmall cell lung cancer. J Cancer Res Ther. 2018; 14(Supplement): S341-S346. doi: 10.4103/0973-1482.235352

48. Chai AWY, Cheung AKL, Dai W, et al. Elevated levels of serum nidogen-2 in esophageal squamous cell carcinoma. Cancer Biomark. 2018; 21(3): 583-590.

49. Li L, Zhang Y, Li N, et al. Nidogen-1: a candidate biomarker for ovarian serous cancer. Jpn J Clin Oncol. 2015; 45(2): 176-82. doi: 10.1093/jjco/ hyul 187

50.Zhou Y, Zhu Y, Fan X, et al. NID1, a new regulator of EMT required for metastasis and chemoresistance of ovarian cancer cells. Oncotarget. 2017; 8(20): 33110-33121. doi: 10.18632/oncotarget.16145

51. Yuan D, Qian H, Guo T, et al. LncRNA-ATB Promotes the Tumourigenesis of Ovarian Cancer via Targeting miR-204-3p. Onco Targets Ther. 2020; 13: 573-583. Published 2020 Jan 20. doi: 10.2147/OTT.S230552
52. Kuk C, Gunawardana CG, Soosaipillai A, et al. Nidogen-2: a new serum biomarker for ovarian cancer. Clin Biochem. 2010; 43(4-5): 355-361. doi: 10.1016/j.clinbiochem.2009.10.012

53. Gupta KK, Gupta VK, Naumann RW. Ovarian cancer: screening and future directions. Int J Gynecol Cancer. 2019; 1: 195-200. doi: 10.1136/ ijgc-2018-000016

54. Bonifácio VDB. Ovarian Cancer Biomarkers: Moving Forward in Early Detection. Adv Exp Med Biol. 2020; 1219: 355-363. doi: 10.1007/9783-030-34025-4_18

55. Muinao T, Deka Boruah HP, Pal M. Diagnostic and Prognostic Biomarkers in ovarian cancer and the potential roles of cancer stem cells - An updated review. Exp Cell Res. 2018; 362(1): 1-10. doi: 10.1016/j. yexcr.2017.10.018

56. Chen $\mathrm{Y}$, Jin $\mathrm{P}$, Zhang HX, et al. Clinical significance of serum nidogen-2 and carbohydrate antigen 125 in patients with ovarian serous cystadenocarcinoma. Tumour. 2011; 31(4): 339-342. doi: 10.3781/j. issn.1000-7431.2011.04.011 